\title{
Weak versus strong wave turbulence in the Majda-McLaughlin-Tabak model
}

\author{
S. Chibbaro, ${ }^{1}$ F. De Lillo, ${ }^{2}$ and M. Onorato ${ }^{2}$ \\ ${ }^{1}$ Sorbonne Université, UPMC Univ Paris 06, CNRS, UMR 7190, Institut Jean Le Rond d'Alembert, \\ F-75005 Paris, France \\ ${ }^{2}$ Dipartimento di Fisica, Università di Torino and INFN, Sezione di Torino, Via P. Giuria 1, Torino 10125, Italy
}

(Received 24 September 2016; published 30 May 2017)

\begin{abstract}
We consider the one-dimensional Majda-McLaughlin-Tabak (MMT) model that describes the interactions of nonlinear and dispersive waves. We perform a detailed numerical study of the direct energy cascade. Our numerical experiments show the following. (i) In the limit of small nonlinearity the spectral slope observed in the statistical steady regime corresponds to the one predicted by the weak-wave-turbulence (WWT) theory. (ii) As the nonlinearity is increased, the WWT theory breaks down and deviations from its predictions are observed. (iii) It is shown that such departures from the WWT theoretical predictions are accompanied by the phenomenon of intermittency, typical of three-dimensional fluid turbulence. Our results clarify the role played by the wave-turbulence theory in the statistical description of nonlinear-dispersive waves described by the MMT model.
\end{abstract}

DOI: 10.1103/PhysRevFluids.2.052603

\section{INTRODUCTION}

Many physical phenomena are associated with the propagation of nonlinear dispersive waves. When the number of degrees of freedom is large enough a statistical description becomes important. The weak-wave-turbulence (WWT) theory is a very general framework by which the statistical properties of a large number of incoherent and interacting waves can be studied. The theory [1-6] is based on a systematic analytical approach that culminates in the so-called wave kinetic equation that describes the evolution of the wave spectrum in time (homogeneity and weak nonlinearity are assumed). The wave kinetic equation is thus the analog of the Boltzmann equation for classical particles and in principle should be able to give reliable predictions for the statistical distribution of energy as a function of wave numbers, as well as for various statistical observables.

The WWT theory has been applied to a variety of fields such as ocean waves [7,8], capillary waves [9,10], Alfvén waves [11], and optical systems [12]. Weak wave turbulence constitutes hence an interdisciplinary tool suitable for investigating the statistical mechanics of a large number of interacting waves. A remarkable aspect of such a theory is that, in the presence of an external forcing and dissipation, exact solutions of the kinetic equation describing constant fluxes of its quadratic conserved quantities can be obtained analytically [3]. Despite the beauty of these theoretical results, it is of paramount importance to verify if the assumptions behind the theory are realized in practice and thus if this approach is suitable to address physical issues of complex wave systems.

A family of one-dimensional nonlinear dispersive wave equations, namely, the MajdaMcLaughlin-Tabak (MMT) model [13], was introduced as an, in principle simple, model for assessing the validity of WWT theory; however, the results reported in [13] were somehow discouraging and it was reported that "the predictions of weak turbulence theory fail and yield to a much flatter spectrum compared with the steeper spectrum observed in the numerical statistical steady state". Deviations from the WWT predictions have been observed numerically in [14] and have been associated with the presence of coherent structures such as quasisolitons [15]. The MMT model has become paradigmlike for the verification of the WWT predictions. Indeed, this model offers the rare opportunity to analyze turbulent dynamics over a large range of scales and it has been proven to display much of the features relevant for turbulent flows [16], even though it is a one-dimensional idealized model. Many studies have been devoted to the understanding of the possible deviations from the mean-field predictions given by wave turbulence. Many numerical 


\section{S. CHIBBARO, F. DE LILLO, AND M. ONORATO}

and experimental observations have pointed out the existence of nontrivial statistics [8,17-19], in numerical experiments [20,21] and also in integrable systems [22]. As in strong turbulence, the understanding of this phenomenon is for now elusive.

\section{MODEL}

In this Rapid Communication we address this issue of intermittency considering the following model:

$$
i \frac{\partial \psi}{\partial t}=\left|\frac{\partial}{\partial x}\right|^{1 / 2} \psi+|\psi|^{2} \psi+\mathcal{F}+\mathcal{D},
$$

where $\psi=\psi(x, t)$ and $|\partial / \partial x|^{1 / 2} \psi \equiv F^{-1}\left[|k|^{1 / 2} F(\psi)\right]$, with $F[\cdots]$ and $F^{-1}[\cdots]$ the direct and inverse Fourier transform operators, respectively. The corresponding linear dispersion relation is of the form $\Omega(k)=\sqrt{|k|}$, which allows for four-wave resonant interactions. In addition, $\mathcal{F}$ and $\mathcal{D}$ are two terms that have been included in order to mimic forcing and dissipation.

The model in (1) belongs to the MMT family of equations. In the absence of forcing and dissipation Eq. (1) preserves the quantity $N=\int|\psi(x)|^{2} d x$ (which we refer to as the number of particles) and the Hamiltonian $H$, which can be written as

$$
H=H_{l i n}+H_{n l}=\int|| \partial /\left.\left.\partial x\right|^{1 / 4} \psi\right|^{2} d x+\frac{1}{2} \int|\psi|^{4} d x .
$$

The Hamiltonian is written as the sum of two terms $H_{l i n}$ and $H_{n l}$ that account for a linear and a nonlinear contribution, respectively. The crucial assumption of WWT is that $\epsilon=H_{n l} / H_{l i n} \ll 1$. This ratio thus constitutes a small parameter that allows the perturbative approach at the basis of the development of the WWT theory.

By imposing a forcing confined at large scales (small $k$ ) and a dissipation at both large and small (large $k$ ) scales, a direct cascade in $k$ space, characterized by a constant flux of linear energy $H_{\text {lin }}$, is predicted by the WWT theory. As in the original work, the dissipation at large scales is added to avoid energy piling up at the lowest mode, since the model supports also an inverse cascade [13]. In the statistically stationary regime, the spectrum is expected to exhibit the power law $n_{k} \sim k^{-1}$, where $n_{k}=\left\langle\left|\psi_{k}\right|^{2}\right\rangle$, with the angular brackets indicating an ensemble average. Verification of such predictions has failed so far [13-15]. In particular, a spectrum of the type $n_{k} \sim k^{-5 / 4}$ has been observed in numerical simulations as a new final statistical steady state, consistently with a different closure proposed on heuristic grounds.

In order to produce a stationary direct cascade, we have performed numerical simulations with a deterministic instability-type forcing, which can be written in Fourier space as

$$
\mathcal{F}_{k}=f \psi_{k}, \quad k \in\left[k_{\min }, k_{\max }\right],
$$

where $f$ is a constant and a dissipation

$$
\mathcal{D}_{k}=\mathcal{D}_{k}^{-}+\mathcal{D}_{k}^{+}=\left(v^{-}|k|^{-m}+v^{+}|k|^{n}\right) \psi_{k},
$$

with $m, n>0 ; v^{-}$and $v^{+}$are constant coefficients.

From Eq. (1) it is possible to establish four different time scales associated with dispersion, nonlinearity, and high- and low-wave-number dissipation:

$$
\begin{aligned}
& \tau_{\text {disp }}=\frac{1}{k_{0}^{1 / 2}}, \quad \tau_{\text {nlin }}=\frac{1}{\left|\psi_{0}\right|^{2}}, \\
& \tau_{\text {diss }}^{+}=\frac{1}{v^{+} k_{0}^{n}}, \quad \tau_{\text {diss }}^{-}=\frac{k_{0}^{m}}{v^{-}},
\end{aligned}
$$

where $k_{0}$ and $\left|\psi_{0}\right|$ are a characteristic wave number and amplitude, respectively. We can then define the following dimensionless parameters as the ratio between the dispersive or dissipative and 
TABLE I. Parameters of the two numerical simulations whose spectra are shown in Fig. 2. The values of the power of the hypo- and hyperviscosity terms are, respectively, $m=8$ and $n=8$.

\begin{tabular}{lcccccc}
\hline \hline Run & $f$ & $k_{\min }$ & $k_{\max }$ & $\epsilon_{0}$ & $\mathrm{Re}_{0}^{+}$ & $\mathrm{Re}_{0}^{-}$ \\
\hline 1 & 0.02 & 4 & 7 & 0.5 & $5 \times 10^{22}$ & $2 \times 10^{-3}$ \\
2 & 0.02 & 4 & 7 & 12 & $5 \times 10^{22}$ & $2 \times 10^{-3}$ \\
\hline \hline
\end{tabular}

nonlinear time scales:

$$
\epsilon_{0}=\frac{\left|\psi_{0}\right|^{2}}{k_{0}^{1 / 2}}, \quad \operatorname{Re}_{0}^{+}=\frac{\left|\psi_{0}\right|^{2}}{v^{+} k_{0}^{n}}, \quad \operatorname{Re}_{0}^{-}=\frac{k_{0}^{m}\left|\psi_{0}\right|^{2}}{v^{-}},
$$

where $\mathrm{Re}^{+}$is the analog of the Reynolds number in fluid mechanics. In terms of these parameters, Eq. (1) takes the form

$$
i \frac{\partial \psi}{\partial t}=\frac{1}{\epsilon_{0}} \mathcal{L} \psi+|\psi|^{2} \psi+\mathcal{F}+\frac{1}{\operatorname{Re}_{0}^{+}} \mathcal{D}^{+}+\frac{1}{\operatorname{Re}_{0}^{-}} \mathcal{D}^{-},
$$

where all the variables and the operators $\mathcal{L}, \mathcal{F}$, and $\mathcal{D}$ are now dimensionless. Given a computational domain, $\epsilon_{0}, \mathrm{Re}_{0}^{ \pm}$, and the forcing amplitude represent our control parameters. In order to extricate the different physical effects, we will keep the forcing and the Reynolds numbers constant and change only $\epsilon_{0}$. The latter parameter indeed controls the ratio between nonlinear and linear energy and therefore represents the key parameter in the WWT theory. Once a simulation has reached a stationary state, we will also monitor the degree of nonlinearity of the asymptotic state by considering the ratio of the nonlinear to the linear Hamiltonian:

$$
\epsilon=\epsilon_{0} \frac{\int|\psi|^{4} d x}{2 \int|| \partial /\left.\left.\partial x\right|^{1 / 4} \psi\right|^{2} d x} .
$$

Note that the relation between $\epsilon_{0}$ and $\epsilon$ calculated when a stationary state has been reached is not obvious; in the next section we will find out that the relation between $\epsilon$ and $\epsilon_{0}$ is quasilinear.

We have solved Eq. (7) through a Strang splitting pseudospectral method. In the unforced and undamped case, the method guarantees a conservation of the number of particles and Hamiltonian with a high degree of accuracy. Simulations have been performed in a periodic box of size $L=2 \pi$ with a number of modes set at $2^{13}$. The time marching is carried out with $\Delta t=0.01$.

The coefficients $\epsilon_{0}$ and $\operatorname{Re}_{0}^{ \pm}$and $f$ are selected and the simulation is run until the total number of particles has reached a stationary state. Among the many simulations performed, for the sake of clarity, we will focus on two choices of parameters identified as runs 1 and 2 in Table I. The two cases differ only in the $\epsilon_{0}$. Note that the values of the Reynolds numbers displayed cannot be related to physical conditions in a straightforward manner; indeed, their value is dictated by the use of the hyper- and hypoviscosity in the dissipation terms whose only role is to act as a sink of energy (particles) in the high- (low-) wave-number region of the spectrum. Concerning the nonlinear parameter $\epsilon_{0}$, its value is quasilinearly dependent on $\epsilon$ (see the inset in Fig. 3), the ratio between the nonlinear to linear Hamiltonian; the latter is a measure of the square of the steepness of the waves. In Fig. 1 the evolution of the number of particles for two numerical simulations is plotted. The figure shows that, after a transient, a statistically stationary state is reached. It is also possible to appreciate that the fluctuations of the number of particles are not small, especially for the more nonlinear case, run 2.

Once a stationary state is reached, the spectra and all the other statistical observables are computed, averaging over time until a satisfactory convergence is reached. Over the same amount of time, the 


\section{S. CHIBBARO, F. DE LILLO, AND M. ONORATO}

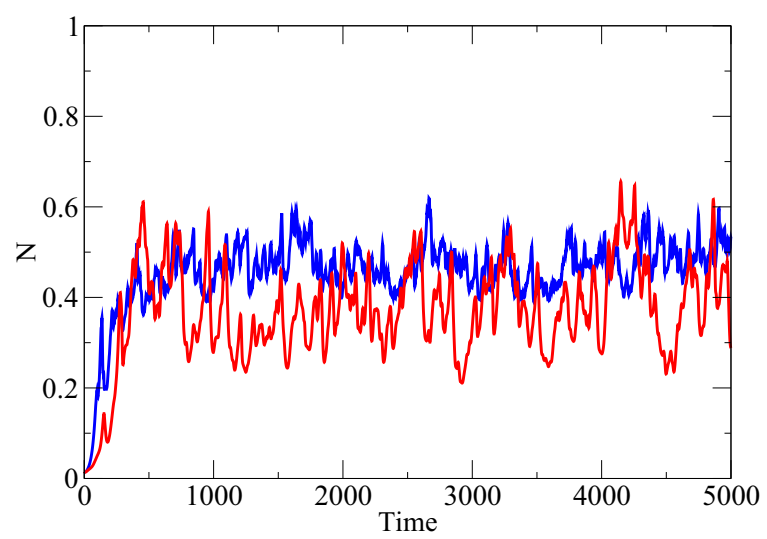

FIG. 1. Evolution of the number of particles for the simulations run 1 (in blue) and run 2 (in red).

averaged value of $\epsilon$ is calculated in order to characterize the degree of nonlinearity of the numerical experiment.

\section{RESULTS}

In Fig. 2 spectra obtained averaging for times larger than 10000 are shown. Both spectra show a power law over a range of about one decade, in the interval $k \in[15,100]$. This scaling is far from both the forcing and the dissipative scales and it corresponds therefore to an inertial range. The figure shows that run 1 , characterized by a lower value of $\epsilon_{0}$, displays a spectral slope that is in agreement with the WWT prediction, while the spectral slope for run 2 appears steeper (see the inset in Fig. 2). Notably, run 2 evidences a statistical steady state in agreement with the MMT spectrum.

In order to appreciate the dependence of the spectral slope $\gamma$ on $\epsilon$ we have performed a number of numerical simulations by keeping $f$ and $\operatorname{Re}_{0}^{ \pm}$constant and changing $\epsilon_{0}$ from 0.1 to 12.5 . In all these cases, a fit is then performed in the inertial range $(k \in[20,100])$. The results are shown in Fig. 3, where the spectral slope $\gamma$ is plotted as a function of $\epsilon$. Interestingly, for $\epsilon \lesssim 0.2$, the slope is in agreement with the prediction of the WWT $\gamma=1$. Increasing $\epsilon$, the slope of the spectrum changes

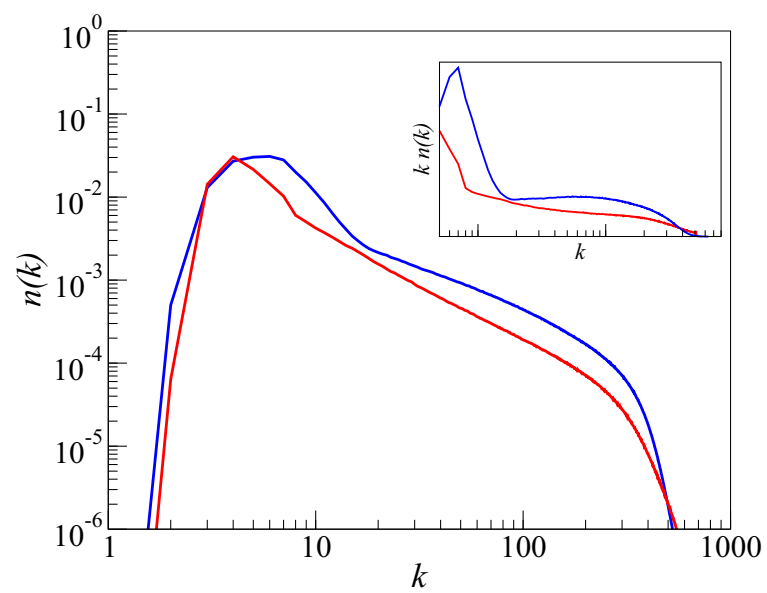

FIG. 2. Spectra of the two numerical simulations whose parameters are reported in Table I. In the inset, the spectra are shown after multiplication by $k$. The plot is on a linear-logarithmic scale. As in all figures, run 1 is blue and run 2 red. 


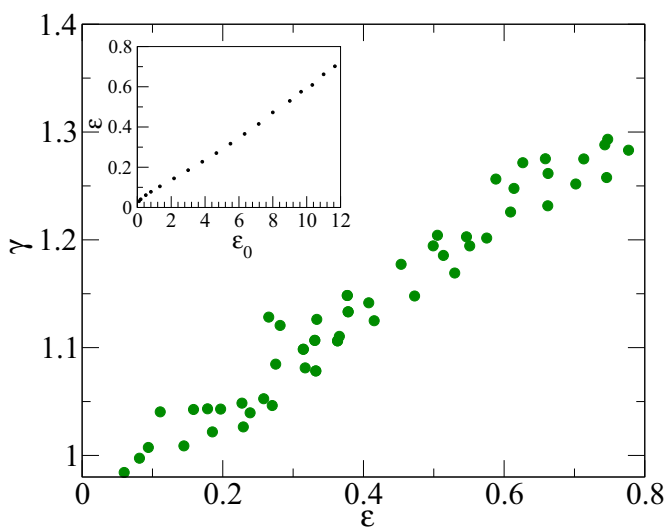

FIG. 3. Slope of the spectrum as a function of the nonlinear parameter $\epsilon$. In the inset $\epsilon$ is plotted as a function of the control parameter $\epsilon_{0}$.

continuously, attaining the MMT prediction $\gamma=5 / 4$ for $\epsilon \gtrsim 0.6$ and reaching steeper slopes for higher values of $\epsilon$. In the inset we show the quasilinear relation between the control parameter $\epsilon_{0}$ and the effective degree of nonlinearity of the simulation $\epsilon$.

In order to look for other signatures of the transition from weak to strong turbulence we compute the quantity $|\psi(k, \omega)|^{2}$, plotted in Fig. 4. It is evident that for run 1 the linear dispersion relation is nicely followed. On the other hand, higher nonlinearity, as in run 2, leads to a broadening of the frequencies at all scales; in such conditions the WWT theory cannot be applied.

As for the velocity field in Navier-Stokes turbulence [23], we consider as the relevant tool for analyzing the intermittent behavior of the wave field the structure functions, defined as

$$
S_{p}(r)=\left\langle|\delta \psi(r)|^{p}\right\rangle=\int|\delta \psi(r)|^{p} \mathcal{P}(\delta \psi) d \delta \psi,
$$

with $\delta \psi=\operatorname{Re}[\psi(x+r)-\psi(x)]$, where $\operatorname{Re}$ denotes the real part. In addition, $\mathcal{P}(\delta \psi)$ is the probability density function (PDF) of the random field $\delta \psi$. For the Wiener-Khinchin theorem, the spectrum is the Fourier transform of the autocorrelation function [23]. Therefore, since the spectral slopes change for different values of $\epsilon$, we may expect that also $S_{2}(r)$ would scale differently as $\epsilon$ changes. Assuming then that $S_{2}(r ; \epsilon)$ scales like $r^{\zeta_{2}(\epsilon)}$ and that the process is self-similar (as we

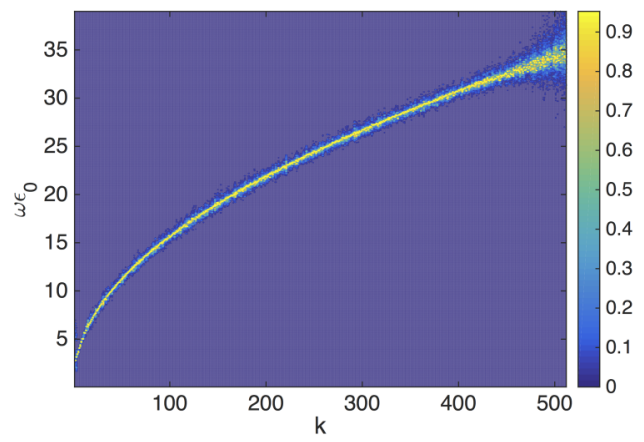

(a)

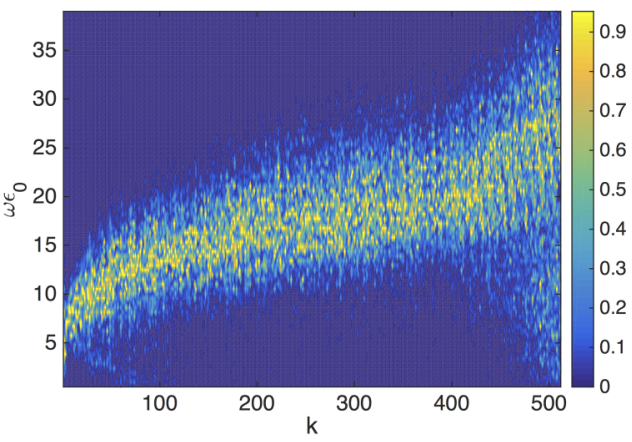

(b)

FIG. 4. Frequency or wave-number spectrum $\left|\psi\left(k, \epsilon_{0} \omega\right)\right|^{2}$ for (a) very small nonlinearity, run 1 , and (b) large nonlinearity, run 2 . For each $k$ the values have been normalized with the maximum in $\omega$ in order to emphasize the shape of the dispersion relation. 


\section{S. CHIBBARO, F. DE LILLO, AND M. ONORATO}

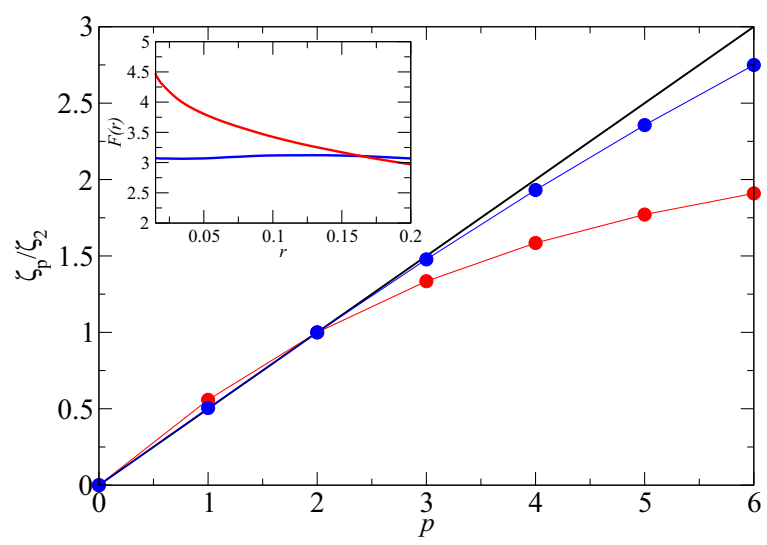

FIG. 5. The ESS scaling exponents $\zeta_{p} / \zeta_{2}$ as a function of $p$ for run 1 (blue) and run 2 (red). The solid line is the prediction for a self-similar process. The inset shows the flatness $S_{4}(r) / S_{2}(r)^{2}$ as a function of $r$.

will see, the latter assumption does not hold for large nonlinearity), i.e., the statistical properties are scale invariant, then we can make the prediction that the relative scaling is universal. In that case, since $S_{p} \sim r^{\zeta_{p}(\epsilon)} \sim S_{2}^{p / 2} \sim r^{(p / 2) \zeta_{2}(\epsilon)}$, we would expect that $\zeta_{p}(\epsilon) / \zeta_{2}(\epsilon)=p / 2$, i.e., $\epsilon$ independent and linear in $p$. However, if the PDF is not self-similar, higher moments do not follow any simple scaling relation with respect to the second one; therefore, we have that $\zeta_{p}(\epsilon) / \zeta_{2}(\epsilon)$ is a nonlinear (possibly $\epsilon$-dependent) function of $p$. In this case, we are in the presence of an anomalous scaling, as is the case in turbulent flows [24]. Since it is difficult to estimate accurately $\zeta_{p}$ from the scaling of $S_{p}(r)$ versus $r$, the most appropriate procedure turns out to be to plot higher-order structure functions versus $S_{2}$ and get directly the relative scaling exponent [this technique is known as the extended self-similarity (ESS) and was developed [25] for moderate Re flows]. It should be stressed that in the case of hydrodynamic turbulence the exact 4/5 law due to Kolmogorov [24,26] implies that the third-order longitudinal structure function of velocity must grow linearly with the separation (in our notation, $\zeta_{3}=1$ ). In that context it is then natural to apply the ESS procedure by computing $\zeta_{p} / \zeta_{3}$. No such law is available for the MMT model, so the choice of the reference moment is here arbitrary.

In Fig. 5 the ratio between $\zeta_{p}$ and $\zeta_{2}$ is shown for the two simulations discussed previously. We observe that, while both curves display a discrepancy with respect to the theoretical prediction, run 2 exhibits a degree of intermittency much larger than run 1 , which is consistent with results obtained in very different wave systems $[8,20,27]$. The nonlinear behavior of $\zeta_{p} / \zeta_{2}$ shown in Fig. 5 implies that for any structure function of order $p>1$, the ratio $S_{p}(r) / S_{2}(r)^{p / 2} \sim r^{\zeta_{p}-p \zeta_{2} / 2}$ increases as $r \rightarrow 0$, or at least down to the dissipation range.

In particular, this is true for the flatness $F=S_{4}(r) / S_{2}^{2}(r)$, which takes the value $F=3$ for a Gaussian field and does not depend on $r$ for any self-similar field. On the other hand, an increasing flatness at small separation indicates that large fluctuations are more frequent at those scales, a phenomenon called intermittency [24]. In particular, the inset of Fig. 5 shows that the $F(r)$ is constant for the weakly nonlinear run 1 (for which $F \approx 3$ as in Gaussian case), while for more nonlinear conditions the flatness is observed to increase up to $\approx 4.5$ at small separations.

The increasing impact of extreme fluctuations at small scales can be better appreciated by inspecting the PDFs $\mathcal{P}(\delta \psi)$ at different separation scale $r$. Figure 6 shows the PDFs of increments, normalized by their standard deviation $\sigma$, for different values of the nonlinearity $\epsilon$. The statistics from run 1 (low nonlinearity) are shown in Fig. 6(a). In this case the PDFs cannot be distinguished from a Gaussian distribution at all separations but at large ones, where they display sub-Gaussian tails. In contrast, Fig. 6(b) shows that run 2 (high nonlinearity) displays strongly non-Gaussian statistics at small scales, with wider and wider tails with decreasing separations. Gaussian statistics are still found at intermediate separations. Again, distributions with sub-Gaussian tails are observed 

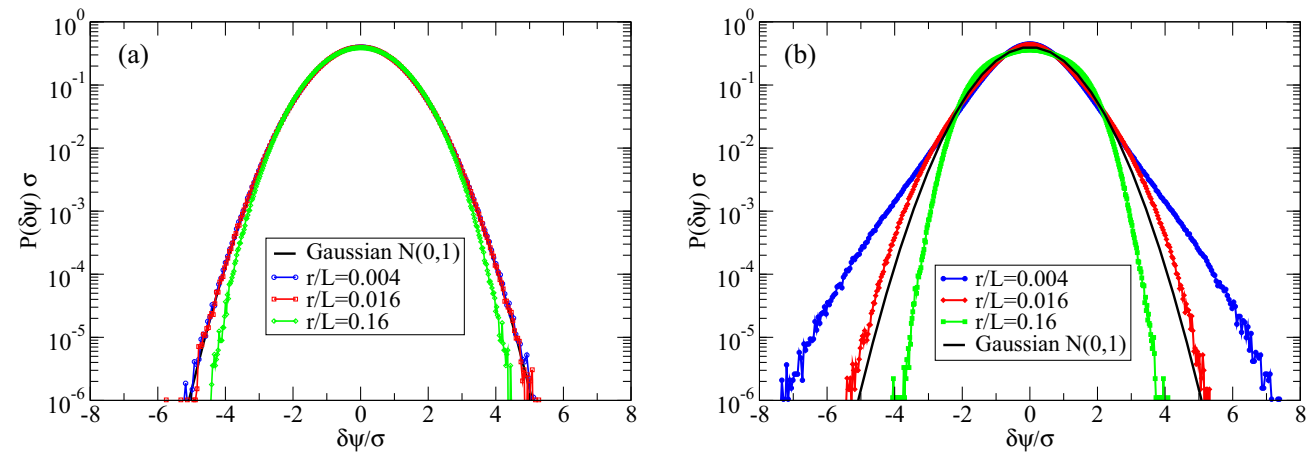

FIG. 6. Probability density function of the increments $\delta \psi$ over different values of the separation $r$ for runs 1 and 2.

at large scales. The scale dependence of the tails of the distributions is indeed the signature of intermittency.

\section{CONCLUSION}

We have addressed the issue of intermittency in wave turbulence considering a paradigmatic model, i.e., the MMT one with $\sqrt{|k|}$ dispersion. Our numerical results indicate that if the nonlinearity is sufficiently small, the statistical steady dynamics is well described by the WWT theory. The important point is that the nonlinear energy must be much smaller than the linear one. In this case, there is no (or very little) sign of intermittency and the WWT theory gives accurate predictions not only for the energy spectrum but also for the whole probability density function of the wave field, which is quasi-Gaussian at all scales. On the other hand, when nonlinearity starts to become large enough, we have shown that the dynamics is even richer than previously depicted, confirming that the MMT model is particularly complex. In particular, we have pointed out that there appears to be a continuous transition from weak to strong turbulence as the nonlinearity is increased. In this case, WWT ceases to represent correctly the system and anomalous scaling is observed, with spectra that differ from the WWT predictions. We have also found out that intermittency is present, with a phenomenology that is similar to that encountered in fluid turbulence. Interestingly, the MMT spectrum is just one of the various possible statistical steady states. A possible explanation of this transition is related to the triggering by nonlinearity of modulational instabilities, which have been found to take place in defocusing MMT $[28,29]$. We expect that our results will motivate and encourage further research and application of wave-turbulence theory. In particular, it would be desirable to include the phenomenon of intermittency in the wave-turbulence formalism.

As a conclusion, we recall that the wave-turbulence theory is the basis for many applications such as the forecasting of ocean waves; the wave kinetic equation (the major outcome of the theory) with forcing (wind) and dissipation (wave breaking) is solved everyday operationally by agencies such as the European Centre for Medium-Range Weather Forecasts or National Oceanic and Atmospheric Administration for predicting the wave periods and heights on a global scale. Therefore, establishing the limitations of the theory is of fundamental relevance for improving the wave forecasting models.

\section{ACKNOWLEDGMENTS}

M.O. was supported by MIUR Grant No. PRIN 2012BFNWZ2. B. Giulinico, D. Proment, and B. Rumpf are acknowledged for fruitful discussions. M.O. acknowledges Gregory Truden from UNITO. We acknowledge support from the European COST Action No. MP1305 "Flowing Matter". Numerical simulations were performed at CINECA via the INFN-FieldTurb grant. 


\section{S. CHIBBARO, F. DE LILLO, AND M. ONORATO}

[1] D. J. Benney and A. C. Newell, Random wave closures, Stud. Appl. Math. 48, 29 (1969).

[2] K. Hasselmann, On the non-linear energy transfer in a gravity-wave spectrum Part 1. General theory, J. Fluid Mech. 12, 481 (1962).

[3] V. E. Zakharov and N. N. Filonenko, Energy spectrum for stochastic oscillations of the surface of a liquid, DOKL AN SSSR 170, 1292 (1966).

[4] G. Falkovich, V. S. Lvov, and V. E. Zakharov, Kolmogorov Spectra of Turbulence (Springer, Berlin, 1992).

[5] S. Nazarenko, Wave Turbulence (Springer, Berlin, 2011), Vol. 825.

[6] A. C. Newell and B. Rumpf, Wave turbulence, Annu. Rev. Fluid Mech. 43, 59 (2011).

[7] G. J. Komen, L. Cavaleri, M. Donelan, K. Hasselmann, H. Hasselmann, and P. A. E. M. Janssen, Dynamics and Modeling of Ocean Waves (Cambridge University Press, Cambridge, 1994).

[8] E. Falcon, S. Fauve, and C. Laroche, Observation of Intermittency in Wave Turbulence, Phys. Rev. Lett. 98, 154501 (2007).

[9] A. N. Pushkarev and V. E. Zakharov, Turbulence of Capillary Waves, Phys. Rev. Lett. 76, 3320 (1996).

[10] C. Falcon, E. Falcon, U. Bortolozzo, and S. Fauve, Capillary wave turbulence on a spherical fluid surface in low gravity, Europhys. Lett. 86, 14002 (2009).

[11] S. Galtier, S. V. Nazarenko, A. C. Newell, and A. Pouquet, A weak turbulence theory for incompressible magnetohydrodynamics, J. Plasma Phys. 63, 447 (2000).

[12] A. Picozzi, J. Garnier, T. Hansson, P. Suret, S. Randoux, G. Millot, and D. N. Christodoulides, Optical wave turbulence: Towards a unified nonequilibrium thermodynamic formulation of statistical nonlinear optics, Phys. Rep. 542, 1 (2014).

[13] A. J. Majda, D. W. McLaughlin, and E. G. Tabak, A one-dimensional model for dispersive wave turbulence, J. Nonlinear Sci. 7, 9 (1997).

[14] V. Zakharov, F. Dias, and A. Pushkarev, One-dimensional wave turbulence, Phys. Rep. 398, 1 (2004).

[15] B. Rumpf, A. C. Newell, and V. E. Zakharov, Turbulent Transfer of Energy by Radiating Pulses, Phys. Rev. Lett. 103, 074502 (2009).

[16] D. Cai, A. J. Majda, D. W. McLaughlin, and E. G. Tabak, Spectral bifurcations in dispersive wave turbulence, Proc. Natl. Acad. Sci. USA 96, 14216 (1999).

[17] E. Falcon, S. Aumaître, C. Falcón, C. Laroche, and S. Fauve, Fluctuations of Energy Flux in Wave Turbulence, Phys. Rev. Lett. 100, 064503 (2008).

[18] S. Nazarenko and S. Lukaschuk, Wave turbulence on water surface, Annu. Rev. Condens. Matter Phys. 7, 61 (2016).

[19] E. Falcon, S. G. Roux, and C. Laroche, On the origin of intermittency in wave turbulence, Europhys. Lett. 90, 34005 (2010).

[20] S. Chibbaro and C. Josserand, Elastic wave-turbulence and intermittency, Phys. Rev. E 94, 011101(R) (2016).

[21] R. Meyrand, S. Galtier, and K. H. Kiyani, Direct Evidence of the Transition from Weak to Strong Magnetohydrodynamic Turbulence, Phys. Rev. Lett. 116, 105002 (2016).

[22] S. Randoux, P. Walczak, M. Onorato, and P. Suret, Intermittency in Integrable Turbulence, Phys. Rev. Lett. 113, 113902 (2014).

[23] A. S. Monin and A. M. Yaglom, Statistical Fluid Mechanics: Mechanics of Turbulence (Dover, New York, 2007).

[24] U. Frisch, Turbulence: The Legacy of A. N. Kolmogorov (Cambridge University Press, Cambridge, 1995).

[25] R. Benzi, S. Ciliberto, R. Tripiccione, C. Baudet, F. Massaioli, and S. Succi, Extended self-similarity in turbulent flows, Phys. Rev. E 48, R29 (1993).

[26] A. N. Kolmogorov, Dissipation of energy in locally isotropic turbulence, Dokl. Akad. Nauk SSSR 32, 16 (1941).

[27] R. Meyrand, K. H. Kiyani, and S. Galtier, Weak magnetohydrodynamic turbulence and intermittency, J. Fluid Mech. 770, R1 (2015).

[28] A. C. Newell, B. Rumpf, and V. E. Zakharov, Spontaneous Breaking of the Spatial Homogeneity Symmetry in Wave Turbulence, Phys. Rev. Lett. 108, 194502 (2012).

[29] B. Rumpf and A. C. Newell, Wave instability under short-wave amplitude modulations, Phys. Lett. A 377, 1260 (2013). 